This item was submitted to Loughborough's Research Repository by the author.

Items in Figshare are protected by copyright, with all rights reserved, unless otherwise indicated.

\title{
Photoelectron spectroscopy of manganese-based Heusler alloys
}

PLEASE CITE THE PUBLISHED VERSION

PUBLISHER

(C) American Physical Society

LICENCE

CC BY-NC-ND 4.0

\section{REPOSITORY RECORD}

Brown, D., Michael D. Cropper, K.H. Bedwell, Martin T. Butterfield, S.J. Guilfoyle, Andrew E.R. Malins, and M. Petty. 2019. "Photoelectron Spectroscopy of Manganese-based Heusler Alloys". figshare.

https://hdl.handle.net/2134/1726. 


\title{
Photoelectron spectroscopy of manganese-based Heusler alloys
}

\author{
D. Brown, M. D. Crapper, ${ }^{*}$ K. H. Bedwell, M. T. Butterfield, S. J. Guilfoyle, A. E. R. Malins, and M. Petty \\ Physics Department, Loughborough University, Loughborough, Leicestershire LE11 3TU, United Kingdom
}

(Received 7 July 1997)

\begin{abstract}
Ultraviolet-photoemission spectroscopy has been employed to investigate the valence-band electronic structures of the magnetic alloys $\mathrm{Co}_{2} \mathrm{MnSn}, \mathrm{Cu}_{2} \mathrm{MnAl}$, and $\mathrm{Pd}_{2} \mathrm{MnSn}$. The valence band of $\mathrm{Co}_{2} \mathrm{MnSn}$ extends to approximately $5 \mathrm{eV}$ below the Fermi level with a high density of states immediately below the Fermi edge. $\mathrm{Cu}_{2} \mathrm{MnAl}$ and $\mathrm{Pd}_{2} \mathrm{MnSn}$ have valence-band widths of 6 and $7 \mathrm{eV}$, respectively, with the highest density of states lying some $3 \mathrm{eV}$ below the Fermi level in each case. Photoemission measurements in the region of the Mn $3 p$ threshold indicate that Mn $3 d$ character extends across the full width of the valence band for all three alloys. For $\mathrm{Pd}_{2} \mathrm{MnSn}$, additional photoemission measurements in the region of the $\mathrm{Pd} 4 d$ Cooper minimum confirm this conclusion. Modulations in photocurrent resulting from $\mathrm{Mn} 3 p$-to- $3 d$ resonances across the valence band of $\mathrm{Co}_{2} \mathrm{MnSn}$ are most intense towards the bottom of the band. This is discussed in terms of possible variations in initial-state hybridization. The experimental photoemission spectra are compared with calculated spectra derived from theoretical band structures. Although there are some differences in shape, the bandwidths of the calculated spectra agree very well with the experimental results. [S0163-1829(98)07003-9]
\end{abstract}

\section{INTRODUCTION}

Although there is a wide and expanding group of intermetallic compounds classified as Heusler alloys ${ }^{1-7}$ the archetypal alloys are those with the general formula $X_{2} \mathrm{MnY}$. $^{1-3}$ These alloys possess the characteristic $L 2_{1}$ crystal structure, comprising four interpenetrating face-centered-cubic sublattices: two occupied by atoms of element $X$, one occupied by Mn atoms, and one occupied by $Y$ atoms. The element $X$ is usually a first-row transition or noble metal such as $\mathrm{Co}, \mathrm{Ni}$, $\mathrm{Cu}, \mathrm{Pd}$, or Pt. Element $Y$ is usually a group-IIIB or -IVB element such as $\mathrm{Al}, \mathrm{In}, \mathrm{Sn}$, or Sb. Interest in these alloys arises from the fact that they are considered to be ideal models for the investigation of localized magnetic behavior. If $X$ is nonmagnetic, then the moment of approximately $4 \mu_{B}$ is entirely confined to the $\mathrm{Mn}$ sublattice. ${ }^{2}$ For the $\mathrm{Co}$ and $\mathrm{Ni}$ alloys, an additional moment is also associated with the $X$ atom sites. ${ }^{3}$

For the ordered alloys, the nearest-neighbor separation of Mn sites is such that there is considered to be negligible direct overlap of the $d$ wave functions. As a consequence, the localized magnetic moments have been interpreted in terms of virtual bound Mn $3 d$ states. ${ }^{8,9}$ However, more recent band-structure calculations by Ishida et al., ${ }^{10-12}$ Williams et al., ${ }^{13}$ and Kubler, Williams, and Sommers ${ }^{14}$ have indicated that the Mn $3 d$ majority spin states form a common hybridized band with the $X d$ states, while the Mn $3 d$ minority spin states are mostly unoccupied. The localized magnetic behavior, therefore, is explained in terms of exclusion of minority spin electrons from Mn sites.

Ultraviolet photoemission spectroscopy employing synchrotron radiation has been widely used to probe the electronic structure of alloys and provides a powerful method for investigating the validity of band-structure calculations. ${ }^{15}$ As the photoionization cross sections for the $s$ and $p$ valence states are comparatively low, the valence-band photoemission spectra of Heusler alloys will be dominated by transitions from the Mn and $X d$ states. ${ }^{16}$ The valence-band spec- trum $S\left(h \nu, E_{B}\right)$ can be described by the expression

$$
\begin{aligned}
S\left(h \nu, E_{B}\right)= & C(h \nu)\left[N_{X} \sigma_{X}(h \nu) D_{X}\left(E_{B}\right)\right. \\
& \left.+N_{\mathrm{Mn}} \sigma_{\mathrm{Mn}}(h \nu) D_{\mathrm{Mn}}\left(E_{B}\right)\right] .
\end{aligned}
$$

The excitation energy and initial-state binding energy are represented by $h \nu$ and $E_{B}$, respectively. $C(h \nu)$ includes factors to account for the photon flux, escape depth, detector efficiency, and reflection and refraction of the photon beam at the sample. ${ }^{17,18}$ Assuming that variations in escape depth and detection efficiency with electron kinetic energy are not significant across the valence band at a given excitation energy, then this can be considered constant. It will not, therefore, affect the shape of the valence-band spectrum. $N_{X}$ and $N_{\text {Mn }}$ represent the fractional contribution of $d$ electrons supplied by each element to the density of states (DOS). The variation in the $X n d$ and $\mathrm{Mr} 3 d$ partial density of states (PDOS) across the valence band is represented by $D_{X}\left(E_{B}\right)$ and $D_{\mathrm{Mn}}\left(E_{B}\right)$, respectively, and the corresponding photoionization cross sections are $\sigma_{X}(h \nu)$ and $\sigma_{\mathrm{Mn}}(h \nu) .{ }^{16}$ Variations in cross section across the band are neglected. Equation (1) also assumes that, in each case, the partial densities of states (PDOS's) $D_{X}\left(E_{B}\right)$ and $D_{\mathrm{Mn}}\left(E_{B}\right)$ are normalized so that the sum across all binding energies is equal to unity.

By utilizing the tunable radiation associated with a synchrotron source, the contribution from $\mathrm{Mn} 3 d$ derived states can be further investigated by means of resonant photoemission $^{19}$ and Cooper minimum effects. ${ }^{20}$ At photon energies across the region of the $3 p$ threshold, photoemission from a $3 d$ state can occur either directly or via promotion of a $p$ electron to an unoccupied $d$ state followed by autoionization. The interference of the direct and indirect processes results in a characteristic modulation in photoemission intensity from regions of the valence band possessing Mn $3 d$ character. The line shape is described theoretically by Fano ${ }^{19}$ and the modulated photocurrent $I_{\bmod }\left(h \nu, E_{B}\right)$ can be expressed as 


$$
\begin{aligned}
I_{\bmod }\left(h \nu, E_{B}\right)= & I_{r}\left(h \nu, E_{B}\right)\left[\frac{\left\{q\left(E_{B}\right)+\varepsilon\left(h \nu, E_{B}\right)\right\}^{2}}{1+\varepsilon\left(h \nu, E_{B}\right)^{2}}\right] \\
& +I_{\mathrm{nr}}\left(h \nu, E_{B}\right),
\end{aligned}
$$

where

$$
\varepsilon\left(h \nu, E_{B}\right)=\frac{2\left[h \nu-h \nu_{0}\left(E_{B}\right)\right]}{\Gamma\left(E_{B}\right)} .
$$

$I_{r}\left(h \nu, E_{B}\right)$ and $I_{\mathrm{nr}}\left(h \nu, E_{B}\right)$ represent the photoemission intensities resulting from direct emission of electrons that do and do not contribute to the resonance, respectively. The Fano parameters that characterize the modulation are the resonance energy $h \nu_{0}\left(E_{B}\right)$, the asymmetry parameter $q\left(E_{B}\right)$, and the spectral width $\Gamma\left(E_{B}\right)$. The shape and intensity of a resonance can be influenced by the degree of initialstate hybridization ${ }^{21-23}$ and so a Fano analysis of resonant line shapes can potentially provide information on the localization of state across the valence band.

For the Heusler alloys containing $4 d$ or $5 d$ metals such as $\mathrm{Pd}$ and Pt, the cancellation of matrix element integrals for transitions to particular final states produces a characteristic minimum in the cross section, the so-called Cooper minimum. ${ }^{20}$ At excitation energies corresponding to the region of the Cooper minimum, the relative contributions from transitions arising from $\mathrm{Mn} 3 d$ derived states will be considerably enhanced. If the $\mathrm{Pd} 4 d$ or $\mathrm{Pt} 5 d$ cross section at the Cooper minimum can be considered negligible in comparison to that for Mn $3 d$, then $\sigma_{X}$ in Eq. (1) can be taken as zero and the spectrum will reflect the shape of the Mn $3 d$ PDOS.

The aim of the research described in this paper is to utilize the methods outlined above to provide information on the valence-band electronic structure of the ferromagnetic Heusler alloys $\mathrm{Co}_{2} \mathrm{MnSn}, \mathrm{Cu}_{2} \mathrm{MnAl}$, and $\mathrm{Pd}_{2} \mathrm{MnSn}$. In particular, the Mn $3 p$-to- $3 d$ resonance and $\mathrm{Pd} 4 d$ Cooper minimum will be employed to investigate the PDOS for the Mn $3 d$ band since it is the Mn sites that carry most of the moment in $\mathrm{Co}_{2} \mathrm{MnSn}$ and all of the moment for $\mathrm{Cu}_{2} \mathrm{MnAl}$ and $\mathrm{Pd}_{2} \mathrm{MnSn}$. The results will be discussed in the context of earlier photoemission studies of Heusler alloys and available band-structure calculations.

\section{EXPERIMENTAL DETAILS}

The alloy specimens were prepared by repeatedly melting the appropriate quantities of the high-purity constituent elements in an argon arc furnace. The samples were then annealed in evacuated quartz ampoules at $1073 \mathrm{~K}$ for $48 \mathrm{~h}$ and quench cooled to produce an ordered Heusler $L 2_{1}$ structure. Specimens were cut to shape using spark erosion and the front face of each sample was polished, initially with $\mathrm{SiC}$ and then with successive grades of diamond paste to a final finish of $1 \mu \mathrm{m}$. Alloys were mounted on a stainless-steel sample holder and the assembly was cleaned by immersion in degreasing solvents.

Photoemission measurements were performed under conditions of ultrahigh vacuum on stations 6.1 (Ref. 24) and 6.2 (Ref. 25) at the Daresbury synchrotron radiation source. Samples were cleaned in situ by scraping the surface with a diamond file mounted on a wobble stick, ensuring that the polished layer was completely removed. The elemental composition of the surface was investigated by Auger electron spectroscopy before and after scraping. An angle-integrated, double-pass cylindrical mirror analyzer was employed with a pass energy of $10 \mathrm{eV}$ to detect and energy sort the emitted electrons. The detector was aligned at $90^{\circ}$ to the incoming photon beam. Photon flux was monitored by measuring the drain current from a tungsten mesh and all photoemission spectra were normalized to this flux.

Photoelectron spectra of the valence-band region of each alloy were obtained in two different modes. Electron energy distribution curves (EDC's) were obtained by detecting a range of electron kinetic energies at constant photon energy. Binding energies were then referenced to the Fermi level $\left(E_{F}\right)$, which was arbitrarily assigned a value of zero. The inelastic contribution was removed from each valence-band spectrum using a Shirley algorithm with three iterations. ${ }^{26}$ The experimental resolution in the photon energy range $40-70 \mathrm{eV}$ was estimated by fitting a Fermi-Dirac distribution convoluted with Gaussians of varying half-width at half maximum (HWHM) to the Fermi edge of the spectra. A reasonable fit was obtained for a Gaussian of $0.2 \mathrm{eV}$ HWHM. Constant initial-state (CIS) spectra were obtained by allowing the photon energy and electron kinetic energy to vary synchronously such that $h \nu-E_{K}$ remained constant. This allowed the photon-energy-dependent photoemission intensity of initial states to be investigated. In order to remove the inelastic contribution, a series of EDC's were obtained in $0.5-\mathrm{eV}$ photon energy increments across the excitation energy range of the CIS spectra. Each EDC was normalized such that the photoemission intensity at a given binding energy was consistent with the appropriate CIS spectrum and the inelastic contribution calculated using a Shirley procedure. A smooth inelastic background was then fitted to the data from the EDC's using a fourth-order polynomial and subtracted from the flux normalized CIS spectrum.

\section{RESULTS AND DISCUSSION}

Auger electron spectra were obtained with the samples at normal incidence to the analyzer and employing a primary beam voltage of $3 \mathrm{kV}$ and a modulation of $3 \mathrm{eV}$ peak to peak. The as-loaded samples showed heavy contamination of the surface by carbonaceous deposits with traces of $\mathrm{O}, \mathrm{S}$, and $\mathrm{Cl}$ also present. The spectra of the scraped alloys, however, showed Auger peaks characteristic of the constituent metals. A residual $\mathrm{C}$ peak was observed in the spectrum of the scraped $\mathrm{Co}$ and $\mathrm{Cu}$ alloys, but a correspondingly small $\mathrm{C}$ peak in the spectrum of the Pd alloy may be masked by the overlapping Pd Auger peaks. All the alloys retained traces of $\mathrm{S}$ and $\mathrm{Cl}$ on the surface even after vigorous scraping. It would seem likely, therefore, that this contamination arises from the segregation of bulk impurities. Quantification was performed by measurement of peak to peak heights for the $L M M$ transitions of $\mathrm{Mn}, \mathrm{Co}$, and $\mathrm{Cu}$ at 589,775 , and $920 \mathrm{eV}$, respectively, the $M N N$ transitions of $\mathrm{Pd}$ and $\mathrm{Sn}$ at 326 and $430 \mathrm{eV}$, respectively, and the $K L L$ transition of $\mathrm{Al}$ at 1396 $\mathrm{eV}$. After correction using literature values for the relative sensitivity factors, ${ }^{27}$ the surface stoichiometry was found to be consistent with the nominal bulk composition within the limits of the associated uncertainties. 


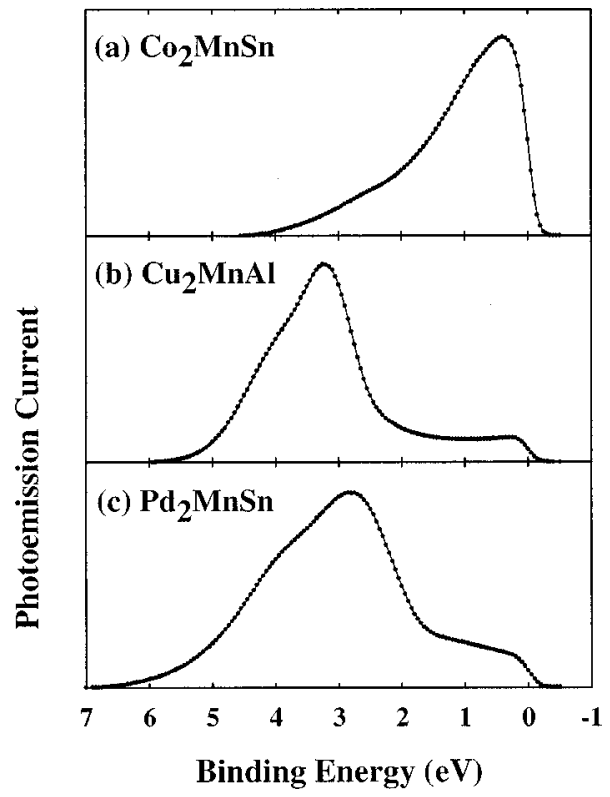

FIG. 1. Electron energy distribution curves for the valence-band region of (a) $\mathrm{Co}_{2} \mathrm{MnSn}$, (b) $\mathrm{Cu}_{2} \mathrm{MnAl}$, and (c) $\mathrm{Pd}_{2} \mathrm{MnSn}$. Spectra for the $\mathrm{Co}$ and $\mathrm{Cu}$ alloys were obtained at an excitation energy of $40 \mathrm{eV}$ in each case. The spectrum for $\mathrm{Pd}_{2} \mathrm{MnSn}$ was obtained at an excitation energy of $70 \mathrm{eV}$. Spectra have been flux normalized and the inelastic contribution removed by means of a Shirley algorithm (Ref. 26).

EDC's across the valence band were initially obtained from different regions of each alloy and at different takeoff angles. However, no discernible differences in spectral shape were observed and all reported spectra were obtained from the center of the sample face at a takeoff angle of $45^{\circ}$. Representative EDC's for each scraped alloy are shown in Fig. 1. Photon energies were selected to minimize the effect of differences in cross section between the $X$ and Mn $d$ states on the spectral shape. The spectra for $\mathrm{Co}_{2} \mathrm{MnSn}$ and $\mathrm{Cu}_{2} \mathrm{MnAl}$ were obtained at a photon energy of $40 \mathrm{eV}$ and the corresponding spectrum for $\mathrm{Pd}_{2} \mathrm{MnSn}$ was obtained at $70 \mathrm{eV}$. As can be seen from Fig. 1(a), the valence band of $\mathrm{Co}_{2} \mathrm{MnSn}$ has a bandwidth of 4-5 eV and is characterized by a high density of states immediately below $E_{F}$. This contrasts with the valence-band spectra of $\mathrm{Cu}_{2} \mathrm{MnAl}$ and $\mathrm{Pd}_{2} \mathrm{MnSn}$ shown in Figs. 1(b) and 1(c), respectively, which reveal the highest occupied density of states to lie approximately $3 \mathrm{eV}$ below $E_{F}$, with only a relatively small shoulder extending to the Fermi edge. The bandwidths of the $\mathrm{Cu}$ and $\mathrm{Pd}$ alloys are also significantly larger, extending 6 and $7 \mathrm{eV}$ below $E_{F}$, respectively. Comparing these results with earlier photoemission measurements on Heusler alloys, it is apparent that the shape of the valence band of $\mathrm{Co}_{2} \mathrm{MnSn}$ is very similar to that of $\mathrm{Ni}_{2} \mathrm{MnSb},{ }^{28}$ while the valence band of $\mathrm{Pd}_{2} \mathrm{MnSn}$ resembles that of $\mathrm{Pd}_{2}$ TiSn. ${ }^{29}$ The spectrum obtained for $\mathrm{Cu}_{2} \mathrm{MnAl}$ has been reported in an earlier paper $^{30}$ and is very similar to that obtained in a separate photoemission study using a He $I(21.2 \mathrm{eV})$ source. $^{31}$

Investigating each alloy in more detail, the Co containing sample will be considered first. Figure 2(a) shows the PDOS for the $3 d$ bands of $\mathrm{Co}_{2} \mathrm{MnSn}$ adapted from the symmetrized augmented-plane-wave (SAPW) calculations of Ishida et al. ${ }^{11}$ To facilitate comparison between experiment and
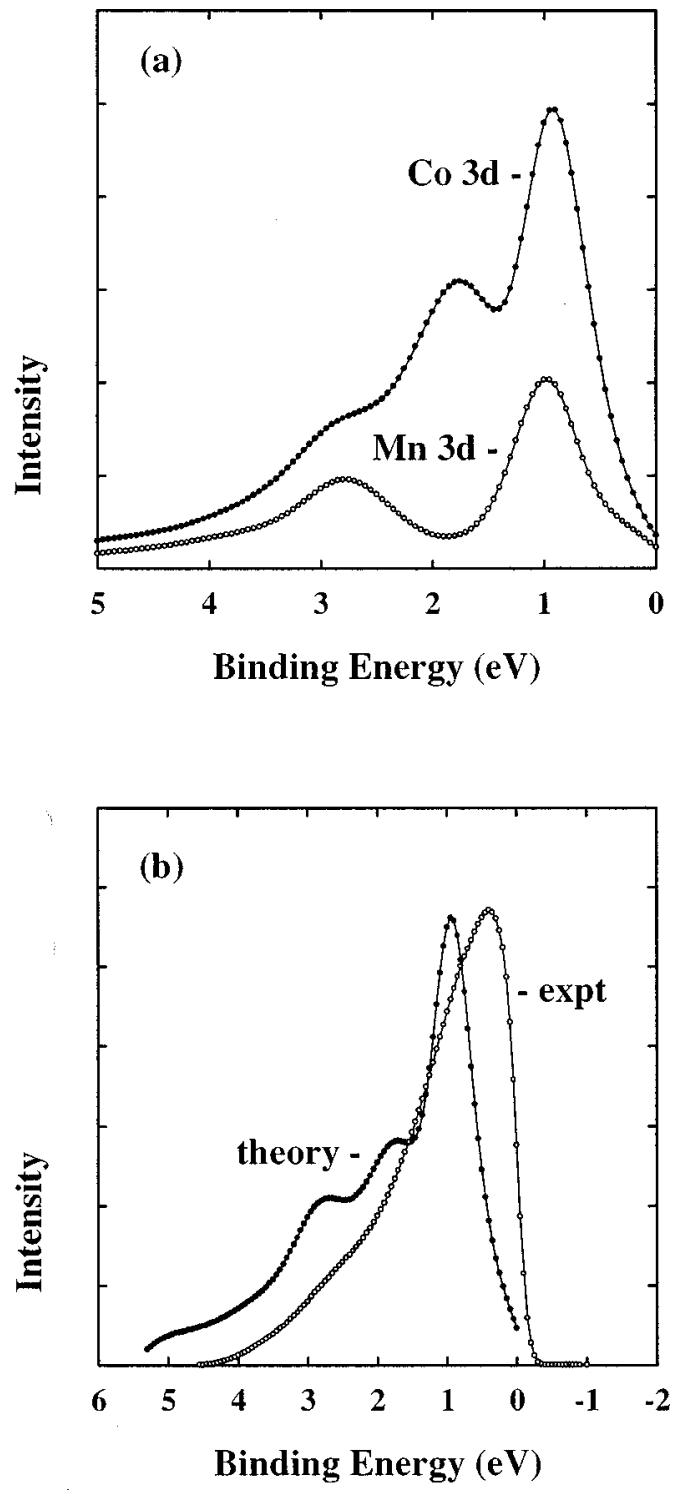

FIG. 2. (a) Partial density of states for the Co and Mn $3 d$ bands in $\mathrm{Co}_{2} \mathrm{MnSn}$ based on the symmetrized augmented-plane-wave calculations of Ishida et al. (Ref. 11) (reproduced with permission). The data have been convoluted to model the effects of intrinsic and extrinsic broadening as described in the text. (b) Comparison of an experimental valence-band spectrum for $\mathrm{Co}_{2} \mathrm{MnSn}$ obtained at an excitation energy of $40 \mathrm{eV}$ with a spectrum derived from the convoluted partial density of states of (a) and the corresponding calculated cross sections (Ref. 16).

theory, the bands have been convoluted to model intrinsic and extrinsic broadening. To model lifetime effects, the bands were convoluted with Lorentzians of HWHM varying in proportion with the square of the binding energy from zero at $E_{F}$ to $0.6 \mathrm{eV}$ at the bottom of the band. ${ }^{30}$ The data were then further convoluted with a Gaussian function of width corresponding to the estimated experimental resolution. The convoluted PDOS were then normalized so that the area under each curve corresponded to the fractional contribution of $d$ electrons supplied by $\mathrm{Co}$ and $\mathrm{Mn}$ to the valence band. The electronic configurations were taken to be $3 d^{8} 4 s^{1}$ for Co and $3 d^{6} 4 s^{1}$ for Mn. ${ }^{11}$ Figure 2(b) shows a comparison of an experimental photoemission spectrum obtained at a photon energy of $40 \mathrm{eV}$ with a spectrum calculated from the 


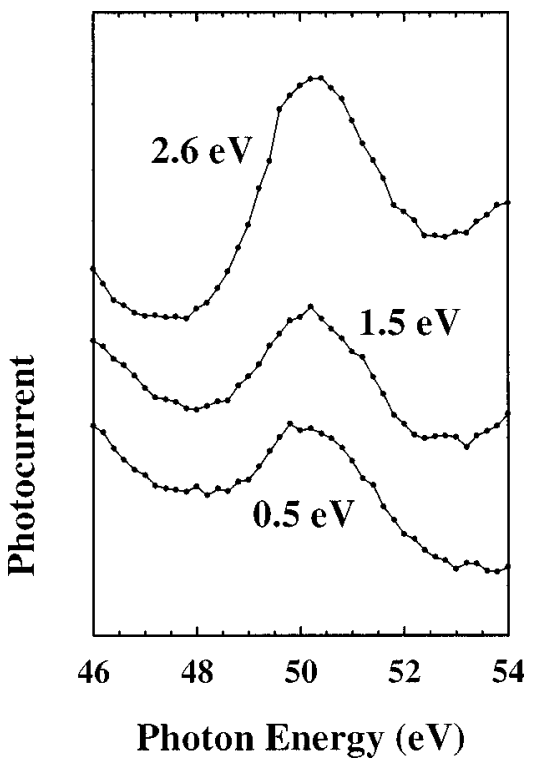

FIG. 3. Constant initial-state spectra for $\mathrm{Co}_{2} \mathrm{MnSn}$ in the region of the Mn $3 p$ threshold. The inelastic contribution was removed in each case as described in the text and the spectra have been arbitrarily displaced along the ordinate for clarity. The corresponding initial-state binding energy is indicated adjacent to each curve.

convoluted PDOS of Fig. 2(a) using Eq. (1). The corresponding photoionization cross sections were obtained from the calculations of Yeh and Lindau. ${ }^{16}$ The calculated and experimental spectra have been arbitrarily normalized so that the area under each curve is identical. Although Eq. (1) does not take into account solid-state effects on the cross sections or self-energy effects, there is reasonable agreement between the experimental and calculated curves in terms of the width and shape of the valence band. The calculated spectrum exhibits three distinct peaks, the most intense at a binding energy of $0.9 \mathrm{eV}$ and peaks of lesser intensity at 1.8 and 2.8 $\mathrm{eV}$. As can be seen from Fig. 2(a), the peaks at 0.9 and 2.8 $\mathrm{eV}$ comprise transitions from degenerate Co and $\mathrm{Mn} d$ states, while the peak at $1.8 \mathrm{eV}$ comprises mainly contributions from states with Co $3 d$ character. The experimental spectrum shows an intense peak with a maximum immediately below $E_{F}$ and a very weak shoulder at approximately $2.6 \mathrm{eV}$. Although the predicted peaks at 1.8 and $2.8 \mathrm{eV}$ in the calculated spectrum are not clearly reproduced in the experimental spectrum, the agreement with theory is reasonable if broadening effects at higher binding energies have been underestimated.

To investigate the contribution of electron states possessing significant $\mathrm{Mn} 3 d$ character to the valence band of $\mathrm{Co}_{2} \mathrm{MnSn}$, CIS spectra were recorded at selected binding energies across the excitation energy range of the Mn $3 p$ threshold. ${ }^{32}$ The inelastic contribution was removed as described in Sec. II and the resulting spectra are shown in Fig. 3. The spectra have been displaced along the ordinate for clarity and the corresponding initial-state binding energies are indicated alongside each curve. Modulations in photocurrent are present at all binding energies, with a maximum at approximately $50 \mathrm{eV}$. Similar modulations have been observed in the CIS spectra of $\mathrm{Ni}_{2} \mathrm{MnSb}$ (Ref. 28) and attributed to Mn $3 p$-to- $3 d$ resonances. The fact that resonant line shapes are observed in all the CIS spectra indicates that $\mathrm{Mn}$


FIG. 4. (a) Spectra obtained on $(50 \mathrm{eV})$ and off $(47 \mathrm{eV})$ the $\mathrm{Mn}$ $3 p$-to-3d resonance for $\mathrm{Co}_{2} \mathrm{MnSn}$. Spectra have been arbitrarily normalized to aid comparison of the change in spectral shape. (b) Comparison of the on-resonance spectrum for $\mathrm{Co}_{2} \mathrm{MnSn}$ with a simulated spectrum. The simulation was obtained by adding the $\mathrm{Mn}$ $3 d$ partial density of states of Fig. 2(a) to the off-resonance spectrum and scaling to match the photoemission intensity of the experimental spectrum at a binding energy of $2.6 \mathrm{eV}$.

$3 d$ character extends across the full width of the valence band. This is in qualitative agreement with the calculated $\mathrm{Mn}$ $3 d$ PDOS of Fig. 2(a). However, it would appear that the intensity of the modulation is enhanced towards the bottom of the band. Figure 4(a) shows the variation in modulation across the valence band more clearly. EDC's were obtained at the resonance maximum $(50 \mathrm{eV})$ and immediately below it $(47 \mathrm{eV})$. In this figure the total intensity of the spectra is not as important as the variation in spectral shape with photon energy. Consequently, the spectra were arbitrarily normalized such that the intensity of the peak just below $E_{F}$ was the same in each case. The difference in shape of the two spectra can be seen quite clearly, with states in the bottom half of the band exhibiting a much stronger resonant signature than those closer to $E_{F}$. Figure 4(b) is a crude attempt to simulate 
an on-resonance spectrum by adding the Mn $3 d$ PDOS of Fig. 2(a) to the off-resonance spectrum and scaling to match the intensity of the experimental on-resonance spectrum at the bottom of the band. This is an identical procedure to that used by other workers. ${ }^{28}$ The simulation will only provide good agreement with experiment if the calculated PDOS is correct and all Mn $3 d$ states modulate uniformly. However, the contributions from states at lower binding energies are oversampled in the simulation, suggesting that the SAPW calculations overestimate the contribution from Mn $3 d$ derived states in the upper part of the band or that the resonant enhancement is correspondingly reduced.

It is possible that differences in resonant intensity could be explained in terms of changes in localization of the Mn $d$ states across the valence band. A similar trend was observed for $\mathrm{Ni}_{2} \mathrm{MnSb}$ by Robey, Hudson, and Kurtz. ${ }^{28}$ Relatively strong resonant features were associated with weakly hybridized states. In the limit of no hybridization, resonances associated with Mn $3 d$ derived states were assumed to be characterized by $q$ values in the range 2.2-2.5, close to those obtained for atomic $\mathrm{Mn} .{ }^{33}$ Weaker resonances, associated with more strongly hybridized states, were found to have $q$ values significantly lower than those for the atomic case. To investigate this aspect further, a Fano analysis was performed on the resonant line shapes of Fig. 3. A nonresonant background was modeled using data from regions of the spectrum on either side of the resonance and Eqs. (2a) and (2b) were used to establish the corresponding Fano parameters. The results are listed in Table I. Backgrounds were modeled in two ways. A fourth-order polynomial was used and also a linear dependence on photon energy was assumed across the region of the modulation. The Fano parameters obtained in the latter case are shown in parentheses and the difference between the two sets of figures provides some indication of experimental uncertainty. Although the absolute values obtained will depend on variations in the removal of the inelastic contribution and on the details of the fitting procedure, general trends can be considered. The resonance energy $h \nu_{0}$ is approximately the same in all cases and is in very good agreement with the values obtained for a similar Fano analysis of the Mn $3 p$-to- $3 d$ resonance in $\mathrm{Ni}_{2} \mathrm{MnSb}$. The asymmetry parameter $q$ increases from a value of $1.7-$ 1.9 close to the Fermi edge to a value of $2.3-2.8$ at the bottom of the band. The value of $q$ obtained at $2.6 \mathrm{eV}$ binding energy is comparable with that obtained for atomic $\mathrm{Mn}^{33}$ A possible explanation, therefore, for the change in $q$ is that the Mn $3 d$ states at the bottom of the band are more localized in nature than those states closer to $E_{F}$. However, other factors apart from the degree of hybridization, such as the presence of multielectron resonant satellites, may affect the valence-band resonance. ${ }^{34,35}$ In addition, overlapping intensity variations due to the appearance of $M V V$ Auger transitions may contribute at the bottom of the band. ${ }^{28}$ Nevertheless, the trend in the resonant photoemission results for $\mathrm{Co}_{2} \mathrm{MnSn}$ is analogous to that obtained for $\mathrm{Ni}_{2} \mathrm{MnSb}$, although for the Ni alloy, $q$ values as low as 0.5 were obtained for resonances arising from initial states immediately below the Fermi edge.

For the case of the $\mathrm{Cu}_{2} \mathrm{MnAl}$ alloy, energy-band calculations have been performed by Ishida et al. employing the same SAPW method utilized in the calculations for
TABLE I. Fano analysis of the Mn $3 p$-to- $3 d$ resonant line shapes for $\mathrm{Co}_{2} \mathrm{MnSn}$. The nonresonant background has been modeled using a polynomial of fourth order in $h \nu$ and also assuming a linear dependence on $h \nu$ across the region of the $3 p$ threshold. Fano parameters obtained from the latter fitting procedure are shown in parentheses.

\begin{tabular}{lccc}
\hline \hline \multirow{2}{*}{$\begin{array}{l}\text { Fano } \\
\text { parameters }\end{array}$} & \multicolumn{3}{c}{ Binding energy $(\mathrm{eV})$} \\
\cline { 2 - 4 }$h \nu_{0}(\mathrm{eV})$ & $49.6(49.5)$ & $49.7(49.6)$ & $49.7(49.7)$ \\
$q$ & $1.9(1.7)$ & $2.3(1.8)$ & $2.8(2.3)$ \\
$\Gamma(\mathrm{eV})$ & $1.8(1.7)$ & $1.9(1.7)$ & $1.6(2.0)$ \\
\hline \hline
\end{tabular}

$\mathrm{Co}_{2} \mathrm{MnSn}$. The electronic configurations were taken as $3 d^{10} 4 s^{1}$ for $\mathrm{Cu}, 3 d^{6} 4 s^{1}$ for $\mathrm{Mn}$, and $3 s^{2} 3 p^{1}$ for Al. ${ }^{10}$ The resulting theoretically derived PDOS's were convoluted and normalized using the same procedure as that outlined for $\mathrm{Co}_{2} \mathrm{MnSn}$. Figure 5 shows a spectrum calculated from Eq. (1) for a photon energy of $40 \mathrm{eV}$ using the theoretical PDOS. At this photon energy $\sigma_{\mathrm{Cu}} / \sigma_{\mathrm{Mn}}$ will be equal to $1.2 .{ }^{16}$ Variations in cross section across the valence band and self-energy effects have not been considered in the calculation. This calculated spectrum is compared with an experimental photoemission spectrum obtained at a photon energy of $40 \mathrm{eV}$. The inelastic background has been removed from the experimental spectrum using a Shirley algorithm and the spectra have been normalized so that the area under the curves is the same in each case. The calculated and experimental spectra provide good agreement in terms of the width of the valence band. In addition, a peak at approximately $3 \mathrm{eV}$ binding energy is observed in both spectra. According to the calculations of Ishida et al., this feature will be dominated by transitions from the filled $\mathrm{Cu} 3 d$ band. However, the calculated and experimental spectra differ markedly in the region 1-2 eV below the Fermi edge. In this region, the calculated spectrum exhibits a second major peak with a maximum at about $1.5 \mathrm{eV}$ binding energy. According to the calculated PDOS, this feature will comprise transitions from both the $\mathrm{Cu}$ and $\mathrm{Mn} 3 d$ bands. In the latter case, the largest contribution to the density of states is located in this region. A very weak maximum at a binding energy of $1.2 \mathrm{eV}$ has been reported for the photoemission spectrum obtained with a $\mathrm{He}$ I source and attributed to the Mn $3 d$ band. ${ }^{31}$ However, in contrast, no evidence has been found for the existence of a comparable feature in the photoemission spectra obtained in this study.

To further investigate the distribution of Mn $3 d$ derived states throughout the valence band of the $\mathrm{Cu}$ alloy, CIS measurements were obtained across the region of the Mn $3 p$ threshold. As for $\mathrm{Co}_{2} \mathrm{MnSn}$, modulations in photocurrent were observed characteristic of a $3 p$-to- $3 d$ resonance. Unlike $\mathrm{Co}_{2} \mathrm{MnSn}$, the intensities of the resonant line shapes did not show any clear variation across the valence band. This fact was confirmed by comparing a background subtracted spectrum obtained off resonance $(47 \mathrm{eV})$ with a spectrum obtained on resonance $(50 \mathrm{eV})$. After arbitrary normalization, the spectra showed very little change in shape. This would tend to suggest that the Mn states are more uniformly distributed across the valence band than the SAPW calculations would suggest, since no correspondingly strong reso- 


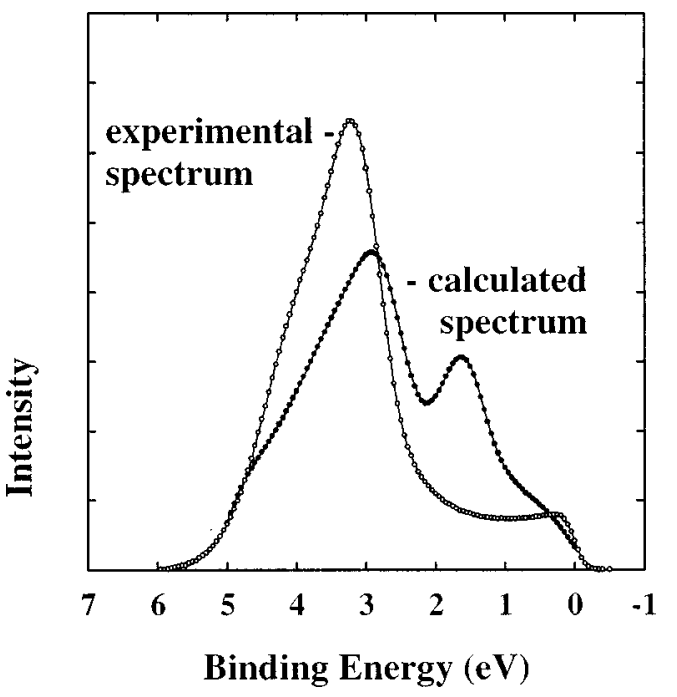

FIG. 5. Comparison of the background subtracted valence-band spectrum for $\mathrm{Cu}_{2} \mathrm{MnAl}$ obtained at a photon energy of $40 \mathrm{eV}$ with a spectrum calculated using Eq. (1) and the symmetrized augmentedplane-wave calculations of Ishida et al. (Ref. 10). The theoretical partial densities of states were convoluted using a procedure identical to that adopted for $\mathrm{Co}_{2} \mathrm{MnSn}$ and corresponding cross sections were taken from the calculated values of Yeh and Lindau (Ref. 16).

nant signature was observed in the region $1-2 \mathrm{eV}$ below $E_{F}$. A Fano analysis of a typical line shape for $\mathrm{Cu}_{2} \mathrm{MnAl}$ is shown in Fig. 6. The data points represent the photoemission intensity obtained after removal of the inelastic background. Fano profiles were obtained by modeling the nonresonant background using quartic (solid curve) and linear (dashed curve) polynomials. The asymmetry parameter $q$ obtained assuming a linear background differs by $30 \%$ from that obtained by modeling the background with a higher-order polynomial. The range of the extracted $q$ values $(1.4-2.0)$ is comparable to the lower range of values obtained for $\mathrm{Co}_{2} \mathrm{MnSn}$ (1.7-1.9).

Considering the Pd alloy in more detail, Fig. 7(a) shows a valence-band photoemission spectrum obtained at a photon energy of $130 \mathrm{eV}$. A comparison with Fig. 1(c) reveals a distinct change in spectral shape from the spectrum obtained at $70 \mathrm{eV}$. The main spectral peak is shifted from a binding energy of $2.9 \mathrm{eV}$ in the $70-\mathrm{eV}$ spectrum to $2.5 \mathrm{eV}$ at $130 \mathrm{eV}$ and is significantly narrower. In addition, a shoulder emerges at $4.4 \mathrm{eV}$ that is not discernible in the lower-excitationenergy spectrum. A spectrum obtained at $180 \mathrm{eV}$, however, possesses a similar shape to that obtained at $70 \mathrm{eV}$. Assuming that differences in sampling depth across this range of excitation energies are not significant and that the cross sections of the Mn $d$ states show a monotonic decrease over the same range, the most likely explanation for the change in spectral shape is a variation in the relative contribution from $\mathrm{Pd} 4 d$ derived states on passing through the Cooper minimum. ${ }^{20}$ If it is assumed that $\sigma_{\mathrm{Pd}}(130 \mathrm{eV})=0,{ }^{36}$ then Fig. 7(a) will reflect the shape of the PDOS for the Mn $3 d$ band in $\mathrm{Pd}_{2} \mathrm{MnSn}$. Similar trends in spectral shape have been observed for the related $\mathrm{Cl}_{b}$ alloy PdMnSb. ${ }^{37}$ In particular, the spectra obtained at photon energies of 70 and $120 \mathrm{eV}$ were similar in shape to Figs. 1(c) and 7(a), respectively. Figure 7(b) shows the calculated $\mathrm{Mn} 3 d$ band for $\mathrm{Pd}_{2} \mathrm{MnSn}$ based on the augmented-spherical-wave ${ }^{14}$ (ASW) and SAPW

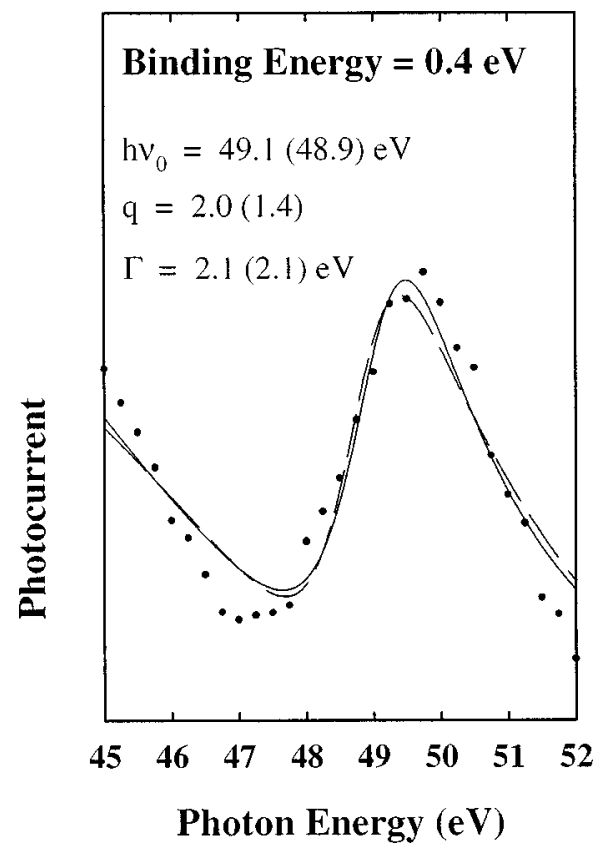

FIG. 6. Constant initial-state spectrum for $\mathrm{Cu}_{2} \mathrm{MnAl}$ obtained at a binding energy of $0.4 \mathrm{eV}$ in the region of the $\mathrm{Mn} 3 p$-to- $3 d$ resonance. Data points represent the photoemission intensity obtained following subtraction of the inelastic background. The curves are Fano line shapes obtained by different fitting procedures. The solid curve was obtained by modeling the nonresonant background with a fourth-order polynomial. The dashed curve was obtained by assuming the nonresonant background to have a linear dependence on photon energy across the region of the resonance. Associated Fano parameters for each curve are indicated in the figure, with the values for the latter fitting procedure shown in parentheses.

methods. ${ }^{12}$ The theoretical data have been convoluted using the same procedure as described for $\mathrm{Co}_{2} \mathrm{MnSn}$ and normalized such that the area under each curve is identical. The $\mathrm{Mn}$ $3 d$ band calculated by the ASW method is in reasonable agreement with the overall shape of the experimental spectrum of Fig. 7(a). However, the sharp peak in the calculated PDOS is shifted closer to $E_{F}$ compared to the empirical PDOS. The SAPW calculation predicts two main peaks in the PDOS at 2.5 and $4.1 \mathrm{eV}$, in close agreement with the position, although not the relative intensity, of the peak and shoulder in the spectrum of Fig. 7(a). The bandwidth predicted by the SAPW method is of the order of $7 \mathrm{eV}$, also in good agreement with the experimental result. However, the comparisons discussed above are only valid if the spectrum at $130 \mathrm{eV}$ is truly representative of the Mn $3 d$ PDOS. This is based on the simplified assumption that all $\mathrm{Pd} 4 d$ states have a negligible contribution at the Cooper minimum. This will not necessarily be the case since the $\mathrm{Pd} 4 d$ states form a band and solid-state effects may be important. ${ }^{17,38,39}$ It is known that bonding and antibonding states in Pd metal exhibit differences in photon-dependent emission intensity, particularly at the Cooper minimum. ${ }^{17}$ If this is also the case for $\mathrm{Pd}$ alloys such as $\mathrm{Pd}_{2} \mathrm{MnSn}$, then the antibonding states close to $E_{F}$ may exhibit a more pronounced atomiclike Cooper minimum than bonding states well below $E_{F}$. Initialstate hybridization also affects the magnitude of the Cooper minimum. ${ }^{17,38,39}$ If, as is suggested by theory, the $\mathrm{Pd} 4 d$ and Mn $3 d$ states form a strongly hybridized, delocalized band, 



FIG. 7. (a) Electron energy distribution curve for $\mathrm{Pd}_{2} \mathrm{MnSn}$ obtained at a photon energy corresponding to the Cooper minimum for Pd $4 d$ photoemission $(130 \mathrm{eV})$. (b) Calculations of the Mn $3 d$ partial density of states for $\mathrm{Pd}_{2} \mathrm{MnSn}$ based on the augmentedspherical-wave (dashed curve) and symmetrized augmented-planewave (solid curve) methods. The convoluted partial densities of states have been adapted from Kubler, Williams, and Sommers (Ref. 14) and Ishida et al. (Ref. 12), respectively, and are reproduced with permission.

then the depth of the Cooper minimum may be significantly reduced from that of the atomic case due to a reduction in $4 d$ character. Such changes have been shown to produce orders of magnitude differences in the Cooper minimum.

Since the EDC obtained at the Pd $4 d$ Cooper minimum does not necessarily convey unambiguous information on the Mn PDOS, CIS spectra were also obtained in the region of the Mn $3 p$-to- $3 d$ resonance. The inelastic contribution was removed as described previously and the results are shown in Fig. 8. It can be seen that modulations in photocurrent are observed at all the binding energies investigated, confirming the presence of Mn $3 d$ derived states across the full width of the valence band. This result is consistent with the assumed PDOS of Fig. 7(a). The relatively weak resonant effect at 0.5 $\mathrm{eV}$ binding energy may reflect a reduced contribution from Mn $3 d$ derived states in the region close to the Fermi edge compared to higher binding energies. This would also be consistent with the assumed shape of the Mn $3 d$ PDOS from Fig. 7(a). Alternatively, however, the weak resonance may be due to strong hybridization of $\mathrm{Mn} 3 d$ states close to $E_{F}$. A comparison of spectral shape for EDC's obtained at photon energies of 47 and $50 \mathrm{eV}$ did not reveal any clear

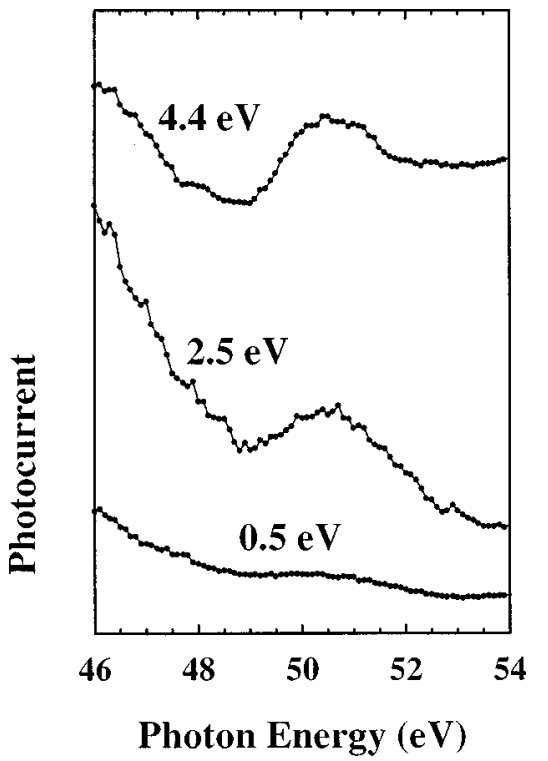

FIG. 8. Constant initial-state spectra obtained across the region of the Mn $3 p$ threshold for $\mathrm{Pd}_{2} \mathrm{MnSn}$. The inelastic contribution has been removed and the spectra have been displaced along the ordinate. Binding energies are indicated alongside the curves.

changes on resonance. Although the Co alloy shows a marked change, the cross section of the Co $3 d$ states is comparable to that of Mn $3 d$ states for photon energies corresponding to the region of the Mn $3 p$ threshold. In the case of the Pd alloy, the Pd $4 d$ cross section is far superior to that for the Mn $3 d$ states. As a result, any changes in spectral shape induced by modulations in photocurrent from $\mathrm{Mn} d$ states may be masked since the spectra will be dominated by transitions from the nonresonating $\mathrm{Pd} 4 d$ band.

To provide more quantitative information on the resonant line shapes at 2.5 and $4.4 \mathrm{eV}$ for $\mathrm{Pd}_{2} \mathrm{MnSn}$, a Fano analysis was performed employing the same procedures as those outlined for $\mathrm{Co}_{2} \mathrm{MnSn}$. The results are given in Table II. The resonance energies are very similar to those obtained for the Co alloy. Although the $q$ values for the Pd alloy exhibit a modest variation with initial-state binding energy, they also appear to show significant variation from the corresponding atomic case. The absolute values of the asymmetry parameters must be interpreted with some caution for reasons already discussed. Nevertheless, the fact that none of the line shapes is characteristic of an atomiclike resonance suggests

TABLE II. Fano analysis of the Mn $3 p$-to- $3 d$ resonant line shapes for $\mathrm{Pd}_{2} \mathrm{MnSn}$. The nonresonant background has been modeled using a polynomial of fourth order in $h \nu$ and also assuming a linear dependence on $h \nu$ across the region of the $3 p$ threshold. Fano parameters obtained from the latter fitting procedure are shown in parentheses.

\begin{tabular}{lcc}
\hline \hline \multirow{2}{*}{$\begin{array}{l}\text { Fano } \\
\text { parameters }\end{array}$} & \multicolumn{2}{c}{ Binding energy $(\mathrm{eV})$} \\
\cline { 2 - 3 }$h \nu_{0}(\mathrm{eV})$ & 2.5 & 4.4 \\
$q$ & $49.5(49.2)$ & $49.6(49.4)$ \\
$\Gamma(\mathrm{eV})$ & $0.6(0.4)$ & $1.0(0.7)$ \\
\hline \hline
\end{tabular}



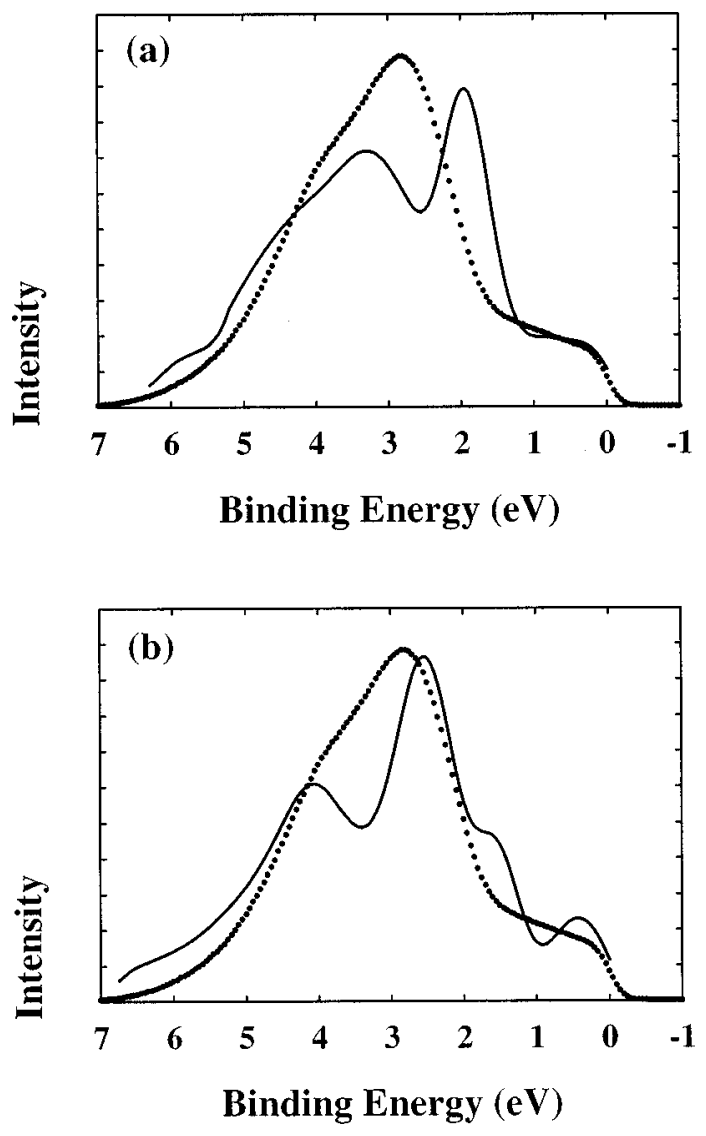

FIG. 9. Comparison of an experimental photoemission spectrum for $\mathrm{Pd}_{2} \mathrm{MnSn}$ obtained at a photon energy of $80 \mathrm{eV}$ with spectra simulated from Eq. (1) and the convoluted partial densities of states calculated by (a) augmented-spherical-wave (Ref. 14) and (b) symmetrized augmented-plane-wave (Ref. 12) methods. For each figure, the experimental spectrum is denoted by closed circles and the calculation is denoted by a solid curve.

that the Mn $d$ states in the Pd alloy may be generally less localized in nature than those of the Co alloy.

Figures 9(a) and 9(b) show an experimental spectrum for $\mathrm{Pd}_{2} \mathrm{MnSn}$ at a photon energy of $80 \mathrm{eV}$ (closed circles) with a spectrum calculated from the theoretical PDOS based on the ASW (Ref. 14) and SAPW (Ref. 12) methods, respectively (solid curves). The electronic configurations of $\mathrm{Pd}$ and $\mathrm{Mn}$ were taken to be $4 d^{10}$ and $3 d^{6} 4 s^{1}$, respectively ${ }^{12}$ and photoionization cross sections were obtained from the calculated values of Yeh and Lindau. ${ }^{16}$ The experimental and calculated spectra have been normalized so that the area under the curves is the same in each case. As for $\mathrm{Co}_{2} \mathrm{MnSn}$ and $\mathrm{Cu}_{2} \mathrm{MnAl}$, the calculations do not take into account solidstate effects on the photoionization cross sections or selfenergy effects. The ASW calculation predicts a DOS with two peaks at 2.0 and $3.3 \mathrm{eV}$. The latter peak comprises mainly contributions from the Pd $d$ states, while the former peak comprises a substantial contribution from the $\mathrm{Mn} d$ states. A comparison with the experimental spectrum suggests that, as was found to be the case for SAPW calculations on $\mathrm{Cu}_{2} \mathrm{MnAl}$, the contribution from $\mathrm{Mn}$ states in the region 1-2 eV below $E_{F}$ is overestimated. The SAPW calculation predicts a DOS with two main peaks at 2.5 and $4.1 \mathrm{eV}$ and a smaller peak immediately below $E_{F}$. The shape of the valence-band spectrum is closely reproduced, particularly if broadening effects have been underestimated. In summary, there are some differences in quantitative detail, but the ASW and SAPW calculations appear to provide reasonable agreement with the experimental valence-band spectrum in terms of the total bandwidth.

\section{CONCLUSIONS}

Ultraviolet photoemission has been employed as an experimental probe of the valence-band structure of the ferromagnetic Heusler alloys $\mathrm{Co}_{2} \mathrm{MnSn}, \mathrm{Cu}_{2} \mathrm{MnAl}$, and $\mathrm{Pd}_{2} \mathrm{MnSn}$. The valence-band spectra of $\mathrm{Co}_{2} \mathrm{MnSn}$ indicate a high density of states immediately below $E_{F}$. The corresponding spectra for $\mathrm{Cu}_{2} \mathrm{MnAl}$ and $\mathrm{Pd}_{2} \mathrm{MnSn}$ indicate a high density of states approximately $3 \mathrm{eV}$ below $E_{F}$. Photoemission spectra have been compared with simulated spectra derived from SAPW (Refs. 10-12) and ASW (Ref. 14) calculations. The experimental spectra will reflect the DOS near the surface of the alloys due to the limited escape depth of the emitted photoelectrons. ${ }^{40}$ This may not necessarily be representative of the bulk DOS. In addition, lifetime broadening and solid-state effects are difficult to account for reliably. Despite these limitations, experiment and theory are in reasonable agreement. The ASW calculation appears to overestimate the density of states at binding energies between 1 and $2 \mathrm{eV}$ below $E_{F}$ for $\mathrm{Pd}_{2} \mathrm{MnSn}$, as does the SAPW calculation for $\mathrm{Cu}_{2} \mathrm{MnAl}$. However, the SAPW calculation reproduces the shape of the experimental spectra quite well for both the Co and Pd alloys. Both methods appear to accurately reproduce the bandwidths.

Cooper minimum and resonant photoemission measurements indicate that Mn $3 d$ character extends across the entire width of the valence band for all the alloys investigated. This is consistent with the idea of completely overlapping, hybridized $d$ states. ${ }^{10-14}$ However, the intensity of the resonant modulation is not uniform in the case of $\mathrm{Co}_{2} \mathrm{MnSn}$, suggesting the possibility of changes in initial-state hybridization across the valence band. States towards the bottom of the band appear to be more localized in nature than those closer to $E_{F}$ and possess Fano line shapes that are characteristic of an atomiclike resonance. These trends in resonant behavior are very similar to those observed for the crystal-field split Mn $d$ band in $\mathrm{Ni}_{2} \mathrm{MnSb}^{28}$ The $t_{2 g}$ states close to $E_{F}$ exhibited only weak resonant behavior, while the $e_{g}$ states at higher binding energies exhibited a much stronger resonance effect. In contrast to $\mathrm{Co}_{2} \mathrm{MnSn}$, the EDC spectra of $\mathrm{Cu}_{2} \mathrm{MnAl}$ and $\mathrm{Pd}_{2} \mathrm{MnSn}$ do not show a clear variation in spectral shape on and off resonance. Fano analysis of the corresponding resonant line shapes in the CIS spectra indicates significant variation in the $q$ values from those expected in the limit of no hybridization. This difference in resonant behavior may be explained by changes in the $X \mathrm{Mn}$ $d$-band hybridization. For the case of the $\mathrm{Cu}$ and $\mathrm{Pd}$ alloys, the Mn $d$ states may be more strongly hybridized with degenerate states from the filled band of the element $X$. For $\mathrm{Co}_{2} \mathrm{MnSn}$, the partially filled Co $d$ band appears to have a high density of states immediately below the Fermi edge and may exhibit a reduced contribution towards the bottom of the band. It has been suggested that a similar reduction in the $\mathrm{Ni}$ 
$3 d$ contribution may account for the increasing localization of $\mathrm{Mn} 3 d$ states at the bottom of the band in $\mathrm{Ni}_{2} \mathrm{MnSb} .{ }^{28}$

To determine the occupancy of the Mn $3 d$ minority spin states, spin-resolved photoemission would need to be employed. ${ }^{41}$ If the minority spin electrons are completely excluded from $\mathrm{Mn}$ sites in $\mathrm{Pd}_{2} \mathrm{MnSn}$ and the contribution from Pd states can be considered negligible at the Cooper minimum, then polarizations close to unity would be observed across the valence-band spectrum at $130 \mathrm{eV}$. However, such measurements would be problematic due to the subambient Curie temperature ${ }^{2}$ and the difficulties of in situ reversable magnetization of the sample.

\section{ACKNOWLEDGMENTS}

The EPSRC funded this research under Grant Nos. 27/ $462,28 / 039$, and 28/155 and also provided financial support for M.T.B. and A.E.R.M. Loughborough University provided support for D.B. Special thanks are due to Professor Kurt Ziebeck for supplying the alloy samples. Stuart Downes and Dave Teehan provided valuable technical support and encouragement at the Daresbury Laboratory.
*Author to whom correspondence should be addressed. FAX: +44 (0) 1509 223986. Electronic address: M.D.Crapper@lboro.ac.uk

${ }^{1}$ F. Heusler, Verh. Dtsch. Phys. Ges. 5, 219 (1903).

${ }^{2}$ P. J. Webster and R. S. Tebble, Philos. Mag. 16, 347 (1967).

${ }^{3}$ P. J. Webster, J. Phys. Chem. Solids 32, 1221 (1971).

${ }^{4}$ Ferromagnetic Materials, edited by E. P. Wohlfarth and K. H. J. Buschow (Elsevier, Amsterdam, 1988), Vol. 4, pp. 287-290, and references therein.

${ }^{5}$ K. U. Neumann, J. Crangle, R. T. Giles, D. Visser, N. K. Zayer, and K. R. A. Ziebeck, Solid State Commun. 84, 577 (1992).

${ }^{6}$ K. U. Neumann, J. Crangle, J. G. Smith, N. K. Zayer, and K. R. A. Ziebeck, J. Magn. Magn. Mater. 140-144, 185 (1995).

${ }^{7}$ M. J. Parsons, J. Crangle, B. Dennis, K. U. Neumann, and K. R. A. Ziebeck, Czech. J. Phys. 46, 2059 (1996).

${ }^{8}$ J. Friedel, Can. J. Phys. 34, 1190 (1956).

${ }^{9}$ P. W. Anderson, Phys. Rev. 124, 41 (1961).

${ }^{10}$ S. Ishida, J. Ishida, S. Asano, and J. Yamashita, J. Phys. Soc. Jpn. 45, 1239 (1978).

${ }^{11}$ S. Ishida, S. Akazawa, Y. Kubo, and J. Ishida, J. Phys. F 12, 1111 (1982).

${ }^{12}$ S. Ishida, Y. Kubo, J. Ishida, and S. Asano, J. Phys. Soc. Jpn. 48, 814 (1980).

${ }^{13}$ A. R. Williams, V. L. Moruzzi, C. D. Gelatt, and J. Kubler, J. Magn. Magn. Mater. 31-34, 88 (1983).

${ }^{14}$ J. Kubler, A. R. Williams, and C. B. Sommers, Phys. Rev. B 28, 1745 (1983).

${ }^{15}$ S. Hufner, Photoelectron Spectroscopy: Principles and Applications, 2nd ed. (Springer-Verlag, Berlin, 1996).

${ }^{16}$ J. J. Yeh and I. Lindau, At. Data Nucl. Data Tables 32, 1 (1985).

${ }^{17}$ G. Rossi, I. Lindau, L. Braicovich, and I. Abbati, Phys. Rev. B 28, 3031 (1983).

${ }^{18}$ B. H. Henke, Phys. Rev. A 6, 94 (1972).

${ }^{19}$ U. Fano, Phys. Rev. 124, 1866 (1961).

${ }^{20}$ J. W. Cooper, Phys. Rev. 128, 681 (1962).

${ }^{21}$ L. C. Davis, Phys. Rev. B 25, 2912 (1982).

${ }^{22}$ A. Kakizaki, K. Sugeno, T. Ishii, H. Sugawara, I. Nagakura, and S. Shin, Phys. Rev. B 28, 1026 (1983).
${ }^{23}$ J. Igarashi and T. Nakano, J. Phys. Soc. Jpn. 55, 1384 (1986).

${ }^{24}$ P. Bailey and F. M. Quinn, SERC Daresbury Laboratory Technical Memorandum No. DI/SCI/TM94E, 1993 (unpublished).

${ }^{25}$ T. S. Turner and D. Teehan, SERC Daresbury Laboratory Technical Memorandum No. DL/SCI/TM85E, 1985 (unpublished).

${ }^{26}$ D. A. Shirley, Phys. Rev. B 5, 4709 (1972).

${ }^{27}$ L. E. Davis, N. C. MacDonald, P. W. Palmberg, G. E. Riach, and R. E. Weber, Handbook of Auger Electron Spectroscopy, 2nd ed. (Physical Electronics Industries Inc., Eden Prairie, 1976).

${ }^{28}$ S. W. Robey, L. T. Hudson, and R. L. Kurtz, Phys. Rev. B 46, 11697 (1992).

${ }^{29}$ D. Brown, M. D. Crapper, K. H. Bedwell, M. Petty, J. G. Smith, and N. D. Telling, J. Phys.: Condens. Matter 9, 2955 (1997).

${ }^{30}$ D. Brown, M. D. Crapper, K. H. Bedwell, L. B. Flannery, M. Petty, and P. A. Skull, J. Phys.: Condens. Matter 8, 5941 (1996).

${ }^{31}$ A. Szytula, T. Czeppe, and J. Stoch, J. Less-Common Met. 159, L5 (1990).

${ }^{32}$ J. C. Fuggle and N. Martensson, J. Electron Spectrosc. Relat. Phenom. 21, 275 (1980).

${ }^{33}$ E. Schmidt, H. Schroder, B. Sonntag, H. Voss, and H. E. Wetzel, J. Phys. B 18, 79 (1985).

${ }^{34}$ D. Chandesris, J. Lecante, and Y. Petroff, Phys. Rev. B 27, 2630 (1983).

${ }^{35}$ M. Iwan, F. J. Himpsel, and D. E. Eastman, Phys. Rev. Lett. 43, 1829 (1979).

${ }^{36}$ H. Wright, P. Weightman, P. T. Andrews, W. Folkerts, C. F. J. Flipse, G. A. Sawatzky, D. Norman, and H. Padmore, Phys. Rev. B 35, 519 (1987).

${ }^{37}$ J. S. Kang, J. G. Park, C. G. Olson, S. J. Yuon, and B. I. Min, J. Phys.: Condens. Matter 7, 3789 (1995).

${ }^{38}$ R. J. Cole, J. A. Evans, L. Duo, A. D. Laine, P. S. Fowles, P. Weightman, G. Mondio, and D. Norman, Phys. Rev. B 46, 3747 (1992).

${ }^{39}$ R. J. Cole, P. Weightman, D. R. Jennison, and E. B. Stechel, J. Electron Spectrosc. Relat. Phenom. 68, 139 (1994).

${ }^{40}$ M. P. Seah and W. A. Dench, Surf. Interface Anal. 1, 2 (1979).

${ }^{41}$ P. D. Johnson, Nucl. Instrum. Methods Phys. Res. A 319, 264 (1992). 